J. Clin. Chem. Clin. Biochem.

Vol. 24, 1986, pp. 379-386

(C) 1986 by Walter de Gruyter \& Co. Berlin - New York

\title{
Eine neue Methode zur schnellen und einfachen Adsorption von Rheumafaktoren aus Serum
}

\author{
Von G. Kolb und R. Allner \\ Zentrallaboratorium (Leiter: Prof. Dr. R. Allner $\dagger)^{1}$ ) der Städtischen Kliniken Fulda, Akademisches Lehrkran- \\ kenhaus der Philipps-Universität Marburg
}

(Eingegangen am 6. August 1985/16. Januar 1986)

Meinem verehrten Lehrer und Mentor $R$. Allner zum Gedenken

Zusammenfassung: Es ist bereits hinreichend bekannt, daß Autoantikörper gegen humanes Gammaglobulin, sogenannte Rheumafaktoren, immunologische Bestimmungsmethoden stören $(1,2)$. Insbesondere die Enzymlinked-Immunoassays (ELISA) zum Nachweis spezifischer Immunität bei frischen viralen Infektionen (z. B. Röteln, Masern etc.) zeigen falsch positive Ergebnisse, wenn die Proben IgM-Rheumafaktoren enthalten $(3,4)$.

Die von uns entwickelte Kombination von Festphase- und Flüssigkeitsphase-Technik - eine Suspension von Glutaraldehyd-vernetztem aggregiertem IgG - stellt eine neue Methode zur schnellen Adsorption und Elimination von Rheumafaktoren aus Serumproben dar.

In einem zweiten Schritt können bei saurem $\mathrm{pH}$ die Rheumafaktoren wieder desorbiert werden, so daß das Adsorptionsmaterial nach Waschen in neutralem Puffer bzw. isotoner $\mathrm{NaCl}$-Lösung erneut verwandt werden kann.

Eine Aḍorption spezifischer Antikörper konnte ausgeschlossen werden.

A new method for rapid adsorption of rheumatoid factor from serum

Summary: It is well known that autoantibodies directed against human $\gamma$-globulin, the so called rheumatoid factor (Rheumafaktor), show interference $(1,2)$ with immunochemical methods. Especially the enzyme-linked immunoassays for detecting the early immune reaction against virus-infections (e. g. rubella, measles, and others) show irregular positive results, if the tests are performed in samples containing IgM rheumatoid factor $(3,4)$.

We describe a new method for the rapid adsorption and elimination of rheumatoid factor from rheumatoid factor containing serum samples, using a suspension of glutaraldehyde-cross-linked, aggregated IgG in a combined solid-and liquid-phase technique. The bound rheumatoid factor can be subsequently desadsorbed at acid $\mathrm{pH}$ so that after washing in neutral-buffer or physiological saline the adsorption-material can be used again.

Specific antibodies were not adsorbed.

1) † 27. Januar 1986

J. Clin. Chem. Clin. Biochem. / Vol. 24, 1986 / No. 6 


\section{Einführung}

Die Rolle des Rheumafaktors als Störquelle bei immunologisch-serologischen Verfahren der medizinischen Diagnostik ist seit langem bekannt. Neben Beeinflussung der Lues-Serologie (5) kennen wir die Interferenz mit Agglutinationstesten auf AustraliaAntigen (6), Cryptococcosis (7), mit diversen Immunfluoreszenztechniken (8) und der passiven Hämagglutination (9) zum Nachweis einer Toxoplasmose, Mononucleose u. a.; Einflüsse auf Methoden zur Erfassung zirkulierender Immunkomplexe sind ebenfalls beschrieben $(10,11)$.

Insbesondere aber die radio- und enzymimmunologischen Verfahren zum Nachweis frisch stattgehabter Virusinfektionen werden durch Rheumafaktoren im Sinne einer falsch positiven Reaktion beeinflußt $(2,3$, 12-15). In Hinblick auf Rheumafaktor-Interferenz besonders eindringlich untersucht ist wegen der mit ihm potentiell verbundenen eugenischen Problemstellung der Rubella-spezifische IgM-Nachweis (1).

Das relativ häufige Vorkommen von Rheumafaktoren auch bei Krankheiten außerhalb des rheumatischen Formenkreises bzw. auch klinisch Gesunden läßt die Gefahr potentieller Fehlbestimmungen durch Rheumafaktor-Interferenz $\mathrm{zu}$ einem nicht vorhersehbaren Risiko weden.

So wird die Bestimmung von spezifischen Antikörpertitern durch das gehäufte gleichzeitige Vorkommen von Rheumafaktoren bei akuten Infektionen mit z. B. Rubella-, Influenza-, Parainfluenza-, Resp.-Syncyteal-, Mumps-, Herpes simplex-Virus u. a. kompliziert (4). Aber auch über den überdurchschnittlich häufigen Nachweis von Autoantikörpern des Rheumafaktor-Types bei Erkrankungen wie Asthma bronchiale $(15,16)$, reaktiver Synovitis (17), degenerativen Gelenkerkrankungen (18), Morbus Basedow (19), der Hepatitis $B$ in akuter und chronischer Verlaufsform $(20,21,22)$, bei lymphoproliferativen Erkrankungen (23) sowie bei Mononucleose (24) und primär und sekundär entzündlichen Erkankungen unterschiedlicher Genese (25) wurde in jüngster Zeit berichtet. Das Auftreten von Rheumafaktoren in der Schwangerschaft $(26,27)$ erhält wegen der möglichen eugenischen Fragestellung zum Beispiel im Zusammenhang mit dem Nachweis einer akuten Rubella-Infektion eine besondere Brisanz und unterstreicht die Wichtigkeit einer suffizienten Rheumafaktor-Adsorption in solchen Fällen.

Die bisher bekannten Rheumafaktor-Adsorptionsmethoden $(1,6,7,28,29)$ weisen eine Reihe von Nachteilen auf, so z. B. begrenzte Bindungskapazität des Adsorptionsmittels $(3,6,7)$ oder Unspezifität der
Adsorptionsphase $(9,30)$. Auch sind Methoden, bei denen der Rheumafaktor als Immunkomplex gebunden in der Probe verbleibt (31), kritisch einzuschätzen.

\section{Material und Methoden}

Proben

1. Seren von 23 Patienten mit und ohne klinisch manifester rheumatoider Arthritis. Die höchste Aktivität an Rheumafaktoren betrug $2,7 \cdot 10^{6} \mathrm{IE} / \mathrm{l}$, die niedrigste $0,017 \cdot 10^{6} \mathrm{IE} / \mathrm{l}$.

2. Anti-Rubella-IgM-haltige Seren $(\mathrm{N}=2)$, Probengewinnung $14 \mathrm{~d}$ nach Aufschießen des Röteln-Exanthems bzw. $14 \mathrm{~d}$ post vaccinationem.

\section{Reagenzien und Test-Kits}

Mittels Ionenaustauschchromatographie (DiethylaminoethylCellulose) isoliertes humanes IgG wurde uns freundlicherweise von der Fa. Biotest, Pharma-GmbH, Dreieich, zur Verfügung gestellt.

Glutaraldehyd reinst, 25\%ig \# E 230/280 - Feinbiochemica der $\mathrm{Fa}$. Serva, Heidelberg.

Phosphatgepufferte Kochsalzlösung der Fa. Behringwerke AG, Marburg.

Rapi-Tex ${ }^{\circledR}-R F$, Behringwerke AG, Marburg.

Cordia ${ }^{\circledR}-R F$, Byk-Mallinckrodt, Dietzenbach.

Röteln-Hämagglutinationshemmtest - HHT, Rube HITं ${ }^{\circledR}$, Behringwerke AG, Marburg.

Enzygnost ${ }^{\circledR}-$ Rubella, Behringwerke AG, Marburg.

Rubazyme ${ }^{\circledR-M}$, Abbott-Diagnostics Div., Irving, Texas, USA.

HAVAB ${ }^{\circledR}-M$ EIA, Abbott Diagnostics Div., Irving, Texas, USA.

Geräte

Quantitative Messungen wurden mit Photometer Eppendorf $1101 \mathrm{M}$ mit Rechner 6432 der Fa. Eppendorf Gerätebau, Hamburg und Photometer "Quantum" mit integriertem Rechner, Fa. Abbott Diagnostics Div., Irving, Texas, USA, durchgeführt.

\section{Allgemeine Analytik}

Quantitative Proteinbestimmungen erfolgten über die BiuretReaktion (32), Reinheitsbeurteilungen (IgG-Präparationen) via Acetatgel-Elektrophorese bzw. mittels Immunelektrophorese; die dazu beutzten Geräte stammten von der Fa: Boskamp Gerätebau KG, Hersel bei Bonn.

Präparation des. Rheumafaktor-Adsorbens: Glutaraldehyd-vernetztes-aggregiertes Ig G

Zur Herstellung des Rheumafaktor-Adsorbens wurde das Prinzip der Vernetzung von Proteinen durch Glutaraldehyd (33) eingesetzt.

$5 \mathrm{ml}$ einer Lösung von $50 \mathrm{~g} / \mathrm{l}$ humanem $\mathrm{IgG}$ in $0,15 \mathrm{~mol} / \mathrm{l}$ Natriumchlorid-Lösung, mit Natrium-Phosphat auf $\mathrm{pH} 7,2$ gepuffert, wurden zur Hitzeaggregation $20 \mathrm{~min}$ bei $60^{\circ} \mathrm{C}$ im Wasserbad inkubiert. Nach Abkühlung auf Raumtemperatur wurde $z u$ dieser Lösung $1 \mathrm{ml}$ einer $0,25 \mathrm{~mol} / 1$ (2,5\%igen) wäßri- 
gen Lösung von Glutaraldehyd unter ständigem Rühren langsam zugetropft. Das sofort bzw. nach wenigen Sekunden entstandene Gel wurde für 3 Stunden bei Raumtemperatur belassen und anschließend durch Spateldruck bereits vorzerkleinert

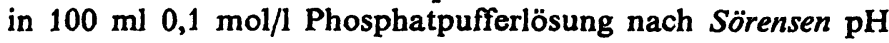
7,2 aufgenommen. Es folgte dann die vollständige Homogenisierung der Gelteilchen in einem (lose passenden) Potter-Homogenisator (Nr. $190 \mathrm{~S}$, Braun, Melsungen) bis zum Erreichen einer guten Suspensionsphase. Es schloB sich eine 15 minütige Zentrifugation bei $10000 \mathrm{~g}$ an. Wiederholung des Homogenisierens und Zentrifugierens bis das Sediment unter dem einfachen Strahl einer Pipette ("Marburg-Pipette“ $=1000 \mu$ l) problemlos und gleichmäßig in Suspension überging. In der Regel war dieser Zustand bereits nach zwei- bis dreimaligem Wiederholen der Prozedur (Homogenisieren und Zentrifugieren) erreicht.

Das Sediment wurde dann in $50 \mathrm{ml} 0,1 \mathrm{~mol} / 1$ Glycin-HClPuffer pH 2,8 überführt und zweimal im gleichen Volumen Glycin-HCl-Puffer gewaschen. Sodann erfolgte dreimaliges Waschen in phosphatgepufferter isotoner $\mathrm{NaCl}-\mathrm{Lösung} \mathrm{pH} 7,2$. Eine Aufbewahrung in phosphatgepufferter isotoner NaCl-Lösung bei $4^{\circ} \mathrm{C}$ ohne Zusatz von Stabilisatoren ist nach unseren bisherigen Erfahrungen ohne Adsorptionskapazitätsverlust über mindestens 9 Monate möglich.

\section{Adsorptionsvorgang, Regeneration des Adsorbens}

Das Verhältnis von Rheumafaktor-Adsorbens (Adsorbens) zu der in den Serumproben enthaltenen Rheumafaktor-Aktivität entscheidet über die Vollständigkeit der Rheumafaktor-Adsorption; je höher der Sedimentanteil der Adsorbens-Suspension, desto höher die Adsorptionsleistung nach Mischen mit einem entsprechend gewählten Serumvolumen. Auf gute Durchmischung der Volumina (Serum und Adsorbenssuspension) muß geachtet werden. Nach 30-minütiger Inkubation bei Raumtemperatur und mehrmaligem zwischenzeitlichen Aufschütteln der Serum-Adsorbens-Puffer-Suspension erfolgt eine Zentrifugation für $5 \mathrm{~min}$ bei $10000 \mathrm{~g}$. Im Sediment befindet sich das Adsorptionsmittel mit adsorbierten Rheumafaktoren. Die so behandelte Probe im Überstand steht dann zur weiteren spezifischen Analytik zur Verfügung.

Durch den Adsorptionsvorgang tritt eine Serumverdünnung ein; wobei das Gesamtsuspensionsvolumen (d. h. Suspensionspuffer + Adsorptionsmittel selbst, da Glutaraldehyd-vernetztaggregiertes Adsorbens als ein Gel wie Puffervolumen zu rechnen ist) die Verdünnung bedingt. Bei den in der vorliegenden Arbeit beschriebenen Versuchen war die genannte Rheumafaktor-Restaktivität nach Adsorption immer auf die Ausgangsaktivität im Ausgangsvolumen zurückbezogen (z. B. nach Rheumafaktor-Adsorption sei die Rheumafaktor-Restaktivitä̆t 0,01 $10^{6} \mathrm{IE} / 1$, Verdünnung durch Adsorptionsvorgang $1: 4$, dann beträgt die tatsächliche Restaktivität beżogen auf das Ausgangsvolumen $\left.0,04 \cdot 10^{6} \mathrm{IE} / \mathrm{l}\right)$. Bei den Experimenten zur Kapazitätsbeurteilung des Rheumafaktor-Adsorbens selbst wurde die adsorbierte Rheumafaktor-Aktivität direkt auf ein Adsorbens-Sediment-Volumen bezogen (z. B. pro $\mu$ l Adsorbens adsorbierte Rheumafaktor-Aktivität in IE).

Zur Desorption der gebundenen Rheumafaktoren wurde das Adsorbens-Sediment, nachdem es zuvor der Adsorption gedient hatte, zunächst zweimal in einem 15 fach größeren Volumen an phosphatgepufferter isotoner $\mathrm{NaCl}$-Lösung zur Entfernung von Serumresten gewaschen. Danach Zugabe eines kleineren Volumens (etwa zweifaches ursprüngliches Serumvolumen) an 0,1 mol/1 Essigsäure pH 2,8 zur Desorption. Nach Zentrifugation für $5 \mathrm{~min}$ bei $10000 \mathrm{~g}$ befindet sich die wieder desorbierte Rheumafaktor-Aktivität im Überstand, wo sie entsprechend nachgewiesen werden kann.

Die vollständige, schnelle Desorption der Rheumafaktoren zur Regeneration des Rheumafaktor-Adsorbens erfolgt durch dreimaliges Waschen des zuvor zur Adsorption benutzten Adsor-

bens-Sediments in einem größeren Volumen (ca. 15-20faches Sedimentvolumen) $0.1 \mathrm{~mol} / 1$ Essigsäure. Nach Äquilibrierung der Adsorbens-Suspension gegen phosphatgepufferte isotone $\mathrm{NaCl}-\mathrm{Lösung} \mathrm{pH} 7.2$ (z. B. durch dreimaliges Waschen in 15 fach größerem Volumen als Adsorbens-Sediment) war die Regeneration beendet. Das Adsorbens stand sodann für einen erneuten Einsatz zur Verfügung.

\section{Ergebnisse und Interpretation der Befunde}

In Abbildung 1 ist beispielhaft der Vorgang einer Rheumafaktor-Adsorption mit nachfolgender Desadsorption und Regeneration des Rheumafaktor-Adsorbens dargestellt. Wie mit der dokumentierten Versuchsdurchführung verdeutlicht, war im adsorbierten Serum gegenüber der noch unadsorbierten Probe (Endtiter $1: 320$ ) keine Rheumafaktor-Aktivität im

\section{Verdünnung}

$$
1: 20
$$

$1: 40$
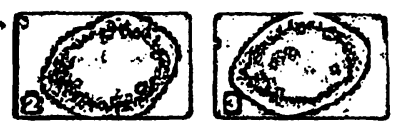

2
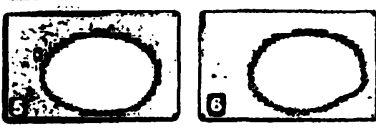

3
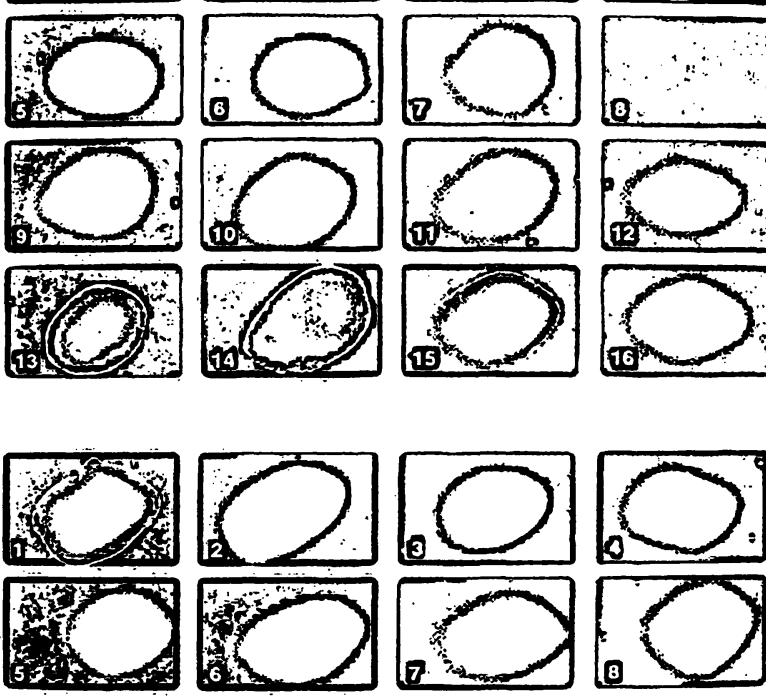

6
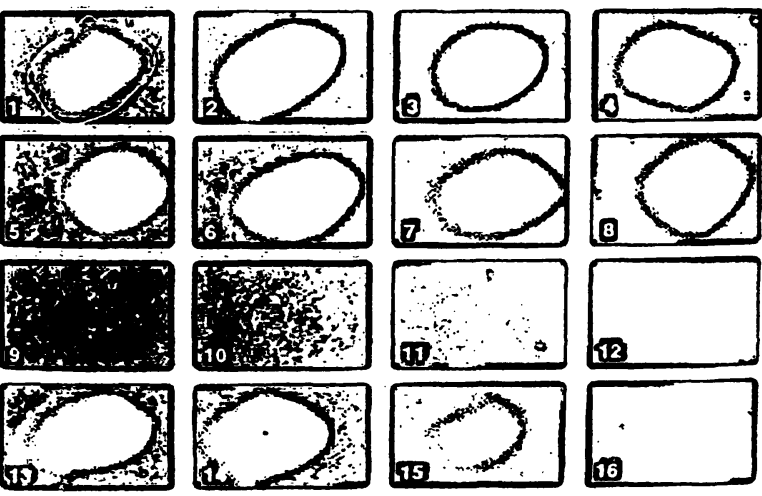

$1: 160$
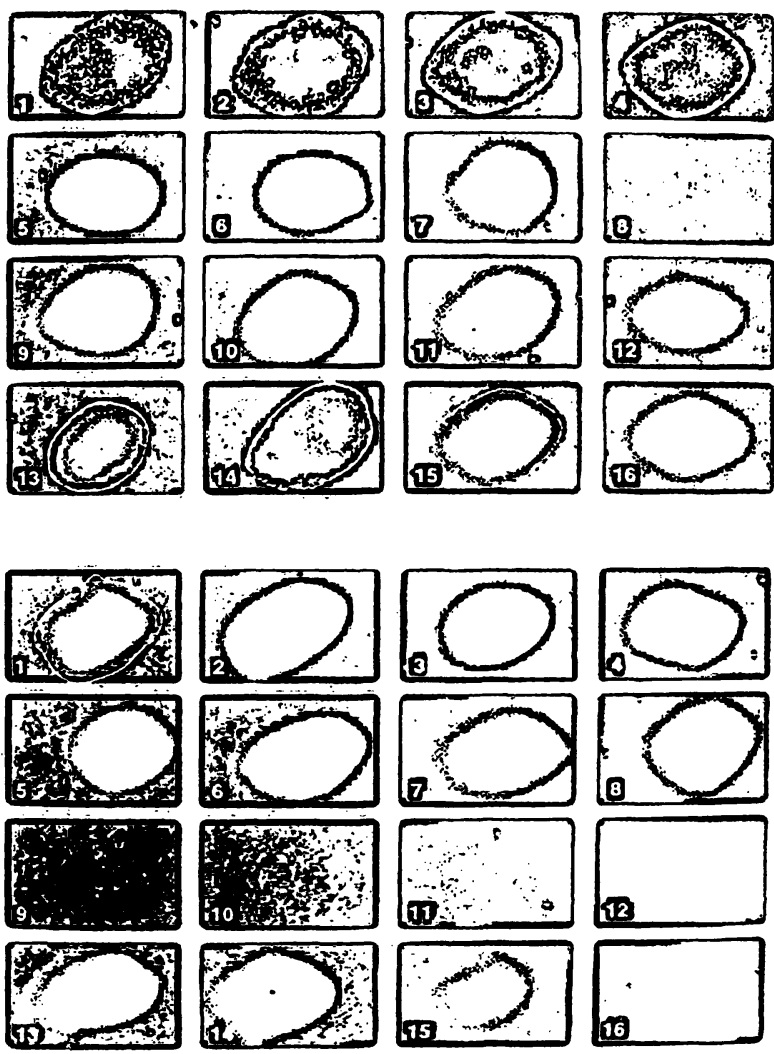
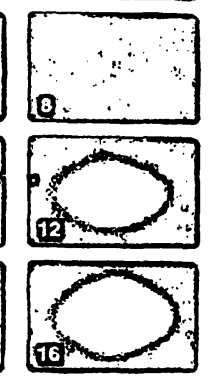

7

Abb. 1. Die Abbildung zeigt einen Adsorptions-Desorptionsvorgang (dokumentiert im Latex-Agglutinationstest); von oben nach unten:

1 = Rheumafaktor-haltiges Serum nicht adsorbiert,

2 = nach Adsorption,

3 = Waschflüssigkeit (phosphatgepufferte isolone $\mathrm{NaCl}-\mathrm{Lösung}$ ) nach Adsorption (es werden durch das Waschen allein keine Rheumafaktoren abgelöst),

$4=$ Desorption mit $0,1 \mathrm{~mol} / \mathrm{l}$ Essigsäure,

$5=2$. Desorption,

$6=3$. Desorption.

7 zeigt nochmalige Adsorption mit demselben Adsorbens wie in 2 nach Regeneration. 
Latex-Agglutinationstest mehr nachweisbar. Die über den Adsorptionsvorgang erreichte Serumverdünnung (1:3) war bei entsprechender ausgleichender Verdünnung der unadsorbierten Kontrolle durch phosphatgepufferte isotone $\mathrm{NaCl}$-Lösung ausgeglichen. Waschen in phosphatgepufferter isotọner $\mathrm{NaCl}$-Lösung alleine ist nicht in der Lage, den gebundenen Rheumafaktor wieder abzulösen. Erst Ansäuerung der Probe - Zugabe von $0.1 \mathrm{~mol} / 1$ Essigsäure zum Adsorbens-Sediment - führt zu seiner Desorption. Nach weiterem Waschen zunächst in $0.1 \mathrm{~mol} / 1$ Essigsäure, dann in phosphatgepufferter isotoner $\mathrm{NaCl}-$ Lösung, steht das Adsorbens zur erneuten Adsorption zur Verfügung und hat, wie ebenfalls in Abbildung 1 dokumentiert, nichts von seiner RheumafaktorBindungskapazität eingebüßt.

Tab. 1. Vergleich von Absorbanz und Rheumafaktor vor und nach Rheumafaktor-Adsorption mittels Rheumafaktor-ELISA (Cordia ${ }^{\circledR}-\mathrm{RF}$ ).

\begin{tabular}{|c|c|c|c|c|}
\hline \multirow[t]{2}{*}{ Patient } & \multicolumn{2}{|c|}{ Unadsorbiert } & \multicolumn{2}{|c|}{ Adsorbiert } \\
\hline & $\mathrm{A}_{405 \mathrm{~nm}}$ & $\begin{array}{l}\text { Rheuma- } \\
\text { faktor } \\
\left(10^{6} \mathrm{IE} / \mathrm{l}\right)\end{array}$ & $\mathrm{A}_{405 \mathrm{~nm}}$ & $\begin{array}{l}\text { Rheuma- } \\
\text { faktor } \\
\left(10^{6} \mathrm{IE} / \mathrm{l}\right)\end{array}$ \\
\hline GAU & 2,4000 & $>0,5$ & 0,166 & 0,015 \\
\hline $\mathrm{TSCH}$ & 1,327 & $>0,5$ & 0,125 & $<0,01$ \\
\hline WEY & 1,228 & $>0,5$ & 0,110 & $<0,01$ \\
\hline$A R N$ & 1,119 & $>0,5$ & 0,107 & $>0,01$ \\
\hline RÜF & 1,068 & $>0,5$ & 0,090 & $>0,01$ \\
\hline WER & 0,269 & 0,04 & 0,028 & 0 \\
\hline KRI* & 0,069 & 0 & 0,047 & 0 \\
\hline neg. Kontr. & 0,070 & 0 & & \\
\hline
\end{tabular}

* Seronegative rheumatoide Arthritis.
Die Effektivität der Rheumafaktor-Adsorption mit der beschriebenen Technik wird an einigen Beispielen aufgezeigt (Tab. 1). Bei einem Mischverhältnis Adsorbensvolumen (Adsorbens-Sediment) mit Serumvolumen wie $1+2$ ließen sich nach einmaliger Adsorption aus den Serumproben bereits total bis subtotal die Rheumafaktoren entfernen, wie anhand der mittels eines Rheumafaktor-ELISA ermittelten Rheumafaktor-Aktivitäten (IE/l) vor und nach Rheumafaktor-Adsorption anhand einiger Serumproben beispielhaft dokumentiert wurde (Tab. 1). Vergleichbare Ergebnisse erhält man auch bei Einsatz der Latex-Ag. glutination (hier nicht gesondert gezeigt).

Zur Untersuchung der Kapazität des RheumafaktorAdsorbens in Abhängigkeit von der jeweiligen individuell vorgegebenen $R$ heumafaktor-Ausgangsaktivität wurden die Rheumafaktor-Aktivitäten von 12 Seren vor und nach Adsorption in einem ELISA auf Rheumafaktor (Cordia ${ }^{\circledR}-\mathrm{RF}$, Byk-Mallinckrodt) bestimmt. Zum Ausgleich der durch Zugabe des Adsorbens erwirkten Serumverdünnung (Verdünnungsfaktor hier $=2$ ) wurden die nach Adsorption ermittelten Rheumafaktor-Werte (IE/l) rechnerisch verdoppelt. Wie in Tabelle 2 ersichtlich, erreichten wir bei dieser Versuchsanordnung Adsorptionsleistüngen von mindestens $60,4 \%$ und maximal $95,3 \%$, gemessen an den jeweiligen Rheumafaktor-Ausgangsaktivitäten. Die mittlere Adsorptionskapazität kann bei den vorgegebenen Versuchsbedingungen mit $76,8 \%$ angegeben werden. Auffällig war, daß keine Korrelation zwischen Höhe der Rheumafaktor-Ausgangșaktivität und Vollständigkeit der Rheumafaktor-Elimination durch die Adsorption besteht. So wurden hohe Rheumafaktor-Aktivitäten teilweise vollständiger adsorbiert als niedrigere.

Tab. 2. Auswertung einer Rheumafaktor-Adsorption an 12 versch. Rheumafaktor-haltigen Seren. Angabe der Adsorptionskapazität in Prozent der Gesamtaktivität des unverdünnten Serums. Verdünnung durch Adsorptionsvorgang wurde durch entsprechende Multiplikation der Rheumafaktor-Restaktivität (nach Adsorption) rechnerisch ausgeglichen.

\begin{tabular}{|c|c|c|c|c|c|}
\hline \multirow[t]{2}{*}{ Patient } & \multirow{2}{*}{$\begin{array}{l}\text { Rheumafaktor } \\
\text { Latex-Titer } \\
\text { vor Adsorption }\end{array}$} & \multicolumn{3}{|c|}{ Rheumafaktoraktivität } & \multirow[b]{2}{*}{ Antêil } \\
\hline & & $\begin{array}{l}\text { vor } \\
\text { Adsorption } \\
\left(10^{6} \mathrm{IE} / \mathrm{l}\right)\end{array}$ & $\begin{array}{l}\text { nach } \\
\text { Adsorption } \\
\left(10^{6} \text { IE/l) }\right.\end{array}$ & $\begin{array}{l}\text { adsorbiert } \\
\left(10^{6} \mathrm{IE} / \mathrm{l}\right)\end{array}$ & \\
\hline WER & $1: 20$ & 0,017 & 0,005 & 0,012 & 0,706 \\
\hline REI & $1: 40$ & 0,072 & 0,019 & 0,053 & 0,742 \\
\hline ARN & $1: 160$ & 0,260 & 0,080 & 0,180 & 0,692 \\
\hline WEJ & $1: 320$ & 0,280 & 0,066 & 0,214 & 0,764 \\
\hline BICK & $1: 160$ & 0,300 & 0,018 & 0,282 & 0,953 \\
\hline SCHNEI & $1: 160$ & 0,360 & 0,110 & 0,250 & 0,694 \\
\hline BLU & $1: 320$ & 0,410 & 0,068 & 0,342 & 0,834 \\
\hline TSCHO & $1: 320$ & 0,440 & 0,078 & 0,362 & 0,823 \\
\hline HER & $1: 160$ & 0,560 & 0,208 & 0,352 & 0,629 \\
\hline $\mathrm{BOH}$ & $1: 160$ & 0,560 & 0,222 & 0,338 & 0,604 \\
\hline GAU & $1: 640$ & 1,040 & 0,170 & 0,870 & 0,837 \\
\hline LAN & $1: 1200$ & 2,700 & 0,160 & 2,540 & 0,941 \\
\hline
\end{tabular}


Da wir bei der Präparation unseres RheumafaktorAdsorbens von der Vorstellung ausgingen, da $\beta$ eine relative Dichte des Gammaglobulin-Substrates, wie wir sie mit Glutaraldehydvernetzung erreichen, eine wichtige Voraussetzung für eine gute Adsorptionskapazität sein müßte, war im folgenden zu klären, ob eine Beziehung auch zwischen der Adsorbens-Suspensionsdichte und der Adsorptionskapazität besteht. Die Klärung dieses Problems war überdies auch im Hinblick auf eine gute (Routine-) handhabbarkeit von nicht unwesentlicher Bedeutung, da dünne Suspensionen (z. B. mit einer Tropfpipette) einfacher Proben zuzusetzen wären.

Die Adsorbens-Suspensionen bzw. die Serumverdünnungen wurden wie folgt erstellt:

\begin{tabular}{lll}
\hline & Adsorbens & $\begin{array}{l}\text { Phosphatgepufferte } \\
\text { isotone NaCl-Lösung } \\
(\mu l)\end{array}$ \\
\hline Suspension a & 80 & 40 \\
Suspension b & 80 & 80 \\
Suspension c & 80 & 320 \\
Suspension d & 80 & 640 \\
\hline
\end{tabular}

Jeweils $100 \mu$ der Suspensionen $a-d$ wurden mit je $100 \mu 1$ Rheumafaktor-Serum vermischt; $d$. h. alle Seren waren durch die Adsorptionsprozedur 1:2 verdünnt, die Verdünnung des Adsorptionsmittels folgte der Reihe $1: 1,5 ; 1: 2 ; 1: 5$ und $1: 9=$ Volumen Adsorbens-Sediment zu Gesamtvolumen (aus Serum, Adsorbens-Sediment und phosphatgepufferter isotoner $\mathrm{NaCl}$-Lösung). Bezieht man dann die adsorbierte Rheumafaktor-Aktivität auf die in der Probe tatsä́chlich vorhandene Menge an Adsorptionsmittel, so erhält man eine deutliche Steigerung der Rheumafaktor-Adsorptionskapazität mit zunehmender Verdünnung der Adșorbens-Suspènsion. Tabelle 3 gibt die Żusammenstellung. Drückt man die gemessenen Kapazitätssteigerungen ab Adsorbens-Ausgangsverdünnung a $(1: 1,5)$ als Zunahme-Faktor aus - Tabelle 4 - so erhält man im Vergleich aller vier Proben (BICK, TSCHO, HER, LAN) ähnliche Steigerungsraten, ausgedrückt dưrch die relative Konstanz der ermịttelten Faktoren.

Tab. 3. Durch $1 \mu$ l Adsorbens adsorbierte Rheumafaktoren (IE) pro $100 \mu \mathrm{l}$ Serum bei Adsorbens-Konzentrationen a-d (vgl. Beschreibung des Versuchsaufbaus).

\begin{tabular}{lrrrr}
\hline Patient & a & b & c & \multicolumn{1}{c}{ d } \\
\hline BICK & 4,3 & 5,7 & 13,6 & 21,1 \\
TSCHO & 5,7 & 7,2 & 16,3 & 21,6 \\
HER & 5,5 & 7,9 & 15,5 & 22,5 \\
LAN & 39,4 & 52,3 & 106,0 & 156,6 \\
\hline
\end{tabular}

Abbildung 2 zeigt das von der Suspensionsdichte abhängige Rheumafaktor-Adsorptionsverhalten unseres Adsorbens in graphischer Form. Dabei demonstriert die untere Kurve (-) die Auftragung der Adsorptionssteigerung bezogen auf die willkürlich gewählte Adsorbens-Stammverdünnung a (Verd. 1,5), die obere Kurve) (--.-) zeigt einen auf die unverdünnte Suspension (reines Sediment) zurückgerechneten (theoretischen) Verlauf als Ausdruck der allgemeingültigen Rheumafaktor-Bindungskinetik unseres

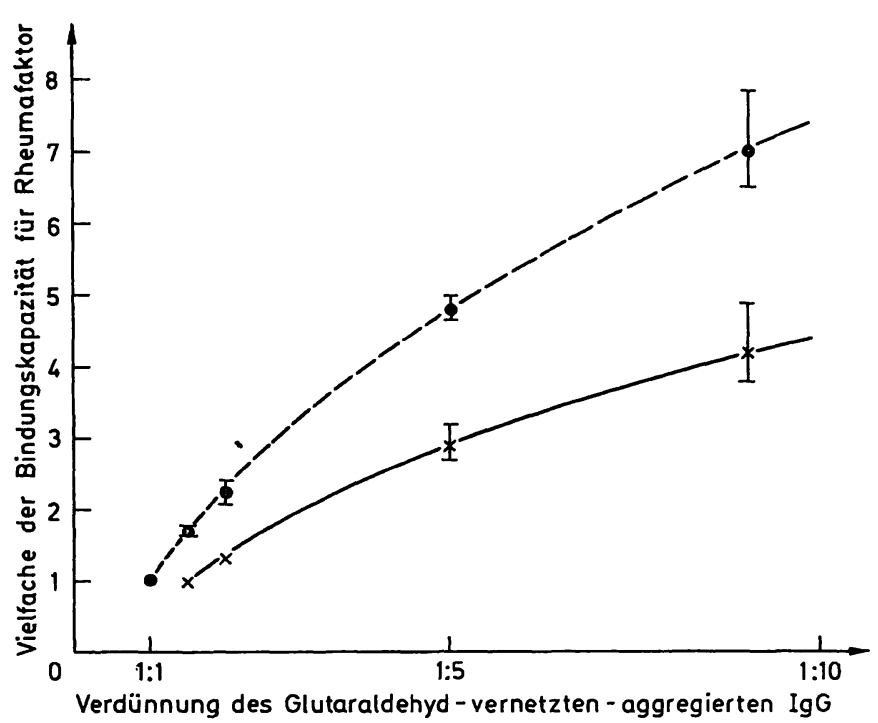

Abb. 2. Abhängigkeit der Rheumafaktor-Adsorptionskapazität von der Suspensionsdichte des Adsorbens - Erläuterungen s. Text. $n=4$; angegeben ist nicht die Standardabweichung, sondern der Bereich zwischen den gemessenen Extremwerten.

\begin{tabular}{llll}
\hline Patient & $\mathrm{A}_{405 \mathrm{~nm}}$ & $\begin{array}{l}\text { Rheuma- } \\
\text { faktor } \\
{\left[10^{6} \mathrm{IE} / \mathrm{l}\right]}\end{array}$ & \\
\hline LAN & 0,511 & 2,70 & Korrelations- \\
GAU & 0,341 & 1,04 & koeffizient \\
BLU & 0,244 & 0,41 & $\mathrm{r}=0,91$ \\
HER & 0,218 & 0,56 & $\mathrm{t}=4,4$ \\
BICK & 0,212 & 0,30 & $0,01<\mathrm{p}<0,02$ \\
BOH & 0,121 & 0,56 & 0 \\
\hline
\end{tabular}

Tab. 4. Zunahme der Rheumafaktor-Adsorptionskapazität von Glutaraldehyd-vernetztem-aggregiertem Immunglobulin ausgedrückt als Faktor bezogen auf die Ausgangsverdünnung $a$.

\begin{tabular}{|c|c|c|c|c|}
\hline Patient & $\mathbf{a}$ & b & c & $d$ \\
\hline $\begin{array}{l}\text { BICK } \\
\text { TSCHO } \\
\text { HER } \\
\text { LAN }\end{array}$ & $\begin{array}{l}1=4,3 \mathrm{IE} \\
1=5,7 \mathrm{IE} \\
1=5,5 \mathrm{IE} \\
1=39,4 \mathrm{IE}\end{array}$ & $\begin{array}{l}1,3 \\
1,3 \\
1,4 \\
1,3\end{array}$ & $\begin{array}{l}3,2 \\
2,9 \\
2,8 \\
2,7\end{array}$ & $\begin{array}{l}4,9 \\
3,8 \\
4,1 \\
4,0\end{array}$ \\
\hline & & $\begin{array}{l}x= \\
1,325\end{array}$ & $\begin{array}{l}x= \\
2,90\end{array}$ & $\begin{array}{l}x= \\
4,20\end{array}$ \\
\hline
\end{tabular}


Adsorbens. Für die weitere Beschreibung der Adsorptionseigenschaften war es wichtig zu klären, ob und inwieweit Lagerungsdauer und/oder Modifikation der Präparation einen Einfluß auf die Adsorptionsleistung des Adsorbens haben.

Es wurden 7 verschiedene Präparationen (Ads. 1-7; Tab. 5) an 4 Rheumafaktor-haltigen Seren getestet. Tabelle 5 gibt den Versuchsaufbau und die nach Adsorption gemessenen Rheumafaktor-Restaktivitäten (IE/l) wieder. „Nativ-acid” bedeutet in diesem $\mathrm{Zu}$ sammenhang, daß das zur Adsorptionspräparation verwandte IgG nicht hitzeaggregiert und damit also nativ mit Glutaraldehyd vernetzt wurde, gleichwohl aber der üblichen Behandlung mit Glycin-HCl-Puffer (,acid“) unterworfen war. „Neutral“ bedeutet: keine Nachbehandlung mit Glycin-HCl. Im Ansatz „Ads. 3 “" wurde erst im Anschluß an die bereits abgeschlossene Präparation des Adsorbens bzw. nach Suspendierung hitzeaggregiert. Alle anderen Chargen unterscheiden sich lediglich durch ihre Herstellungsdaten und damit in der Lagerungsdauer; Zeitpunkt des dokumentierten Versuchs: 01. 07. 1983.

Bei Betrachtung der in Tabelle 5 zusammengefaßten Ergebnisse zeigte die Adsorbenspräparation, bei der die Hitzeaggregation nach abgeschlossener Suspendierung des Glutaraldehyd-vernetzten Immunglobulins erfolgte, die mit Abstand schlechtesten Adsorp- tionsleistungen; offensichtlich verändert Hitzebehandlung nach erfolgter IgG-Vernetzung mittels Glutaraldehyd die Antigenität zuungunsten der Affinität des Rheumafaktor ihm gegenüber. Umgekehrt schafft alleinige Vernetzung von IgG durch Glutaraldehyd ohne vorherige Hitzeaggregation ((Ads. 1) ausreichend dicht beieinanderliegende antigene Determinanten zur stabilen Bindung des Rheumafaktors. Die Lagerungszeit hatte bisher keinen Einfluß auf die Adsorptionsleistung.

Die Inkubationşzeit der Adsorptionsreaktion hat auf Bindung der Rheumafaktoren einen nachweisbaren Einfluß. Eine Inkubationsdauer von 30 Minuten erwies sich unter den Verfahrensbedingungen im Mittel am wirkungsvollsten, gemessen an in den Proben jeweils verbleibenden Rheumafaktor-Restmengen (vgl. Tab. 6). Längere Inkubationszeiten brachten keinen zusätzlichen Effekt. Eine Inkubation von lediglich 10 Minuten wies eine deutlich schlechtere Adsorptionsleistung auf. Absolut unbefriedigende $\mathrm{Er}$ gebnisse zeigte eine $14 \mathrm{~h}$ lange Inkubation bei $4^{\circ} \mathrm{C}$ (Kühlschrank). Eine Zunahme der RheumafaktorAktivität durch einfaches Belassen der nativen Seren für $6 \mathrm{~h}$ bei Raumtemperatur war, wenn auch in relativ geringem Maße, bei zwei der drei Proben feststellbar - Dissoziationsphänomene (34) spielen dabei eventuell eine Rolle.

Tab. 5. Dargestellt ist die Adsorptionskapazität diverser Chargen und Präparationsmodifikationen von Glutaraldehyd-vernetztenaggregiertem-IgM: Ads. 1-7 über die Angabe der jeweiligen Rheumafaktor-Restaktivität nach Adsorption. Der dokumentierte Versuch wurde am 1.7. 83 durchgeführt. Das reine Adsorbens-Sediment war im :Volumenverhältnis $1: 2$ in phosphatgepufferter isotoner $\mathrm{NaCl}$-Lösung suspendiert. $100 \mathrm{ml}$ dieser Suspension wurden $100 \mu \mathrm{l}$ Serum zugefügt. Daraus resultierte eine Serumverdünnung von $1: 2$ und eine Verdünnung des Adsorbens wie $1: 4$.

\begin{tabular}{|c|c|c|c|c|c|c|c|c|c|}
\hline & vor & Ads. & Ads. 1 & Ads. 2 & Ads. 3 & Ads. 4 & Ads. 5 & Ads. 6 & Ads. 7 \\
\hline LAN & & 2,700 & 0,146 & 0,156 & 1,400 & 0,380 & 0,130 & 0,104 & 0,150 \\
\hline TSCHO & & 0,500 & 0,096 & 0,102 & 0,220 & 0,100 & 0,074 & 0,062 & 0,078 \\
\hline BICK & & 0,270 & 0,018 & 0,144 & 0,120 & 0,022 & 0,010 & 0,012 & 0,013 \\
\hline HER & & 0,630 & 0,140 & 0,126 & 0,350 & 0,160 & 0,120 & 0,144 & 0,190 \\
\hline
\end{tabular}

Ads. 1 Adsorbens - nativ-acid

Ads. 2 Adsorbens - neutral

Ads. 3 Adsorbens - nach Vernetzung hitzeaggregiert

Ads. 4 Adsorbens

Ads. 5 Adsorbens

Ads. 6 Adsorbens

Ads. 7 Adsorbens (vom 10. 2. 83)

(vom 23. 1.83)

bei $60^{\circ} \mathrm{C} 20 \mathrm{~min}$ (vom 9.6.83)

(vom 29. 12. 82)

(vom Okt. 82)

(vom 14. 6.83)

(vom 10.6. 83)

Tab. 6. Einfluß der Inkubationszeit auf die Adsorptionskapazität von Glutaraldehyd-vernetztem-aggregiertem-IgM bei Raumtemperatur. Angegeben sind Rheumafaktor-Restaktivitäten in 109 $\mathrm{IE} / 1$. Adsorbens: Serum wie 1:4 (Ads.-Vol.: Ges.-Vol. = 1:4).

\begin{tabular}{|c|c|c|c|c|c|c|c|}
\hline \multirow[t]{2}{*}{ Patient } & \multicolumn{4}{|c|}{ Vor Adsorption } & \multicolumn{3}{|c|}{ Nach Adsorption } \\
\hline & $0 \min$ & $6 \mathrm{~h}$ & $10 \min$ & $30 \mathrm{~min}$ & $2 \mathrm{~h}$ & $6 \mathrm{~h}$ & $14 \mathrm{~h}$ \\
\hline $\begin{array}{l}\text { ARN } \\
\text { BOH } \\
\text { LAN }\end{array}$ & $\begin{array}{l}0,300 \\
0,530 \\
2,700\end{array}$ & $\begin{array}{l}0,320 \\
0,530 \\
3,100\end{array}$ & $\begin{array}{l}0,114 \\
0,220 \\
0,140\end{array}$ & $\begin{array}{l}0,094 \\
0,186 \\
0,110\end{array}$ & $\begin{array}{l}0,082 \\
0,180 \\
0,130\end{array}$ & $\begin{array}{r}0,068 \\
0,164 \\
0,152\end{array}$ & $\begin{array}{l}0,084 \\
0,380 \\
0,520\end{array}$ \\
\hline
\end{tabular}




\section{Hinweise für die praktische Nutzung}

Auf die Beeinflussung immunologischer Bestimmungsverfahren durch entsprechende Rheumafaktor-Aktivität wurde eingangs bereits hingewiesen. Die Gefährlichkeit der Konstellation: Spezifisches IgG bei gleichzeitigem Vorhandensein von IgM-Rheumafaktor hinsichtlich falsch positiver spezifischer IgM-Titer stellt wegen eventueller eugenischer Konsequenzen bei den ELISA zum Nachweis einer frischen RötelnInfektion einen besonderen Problemfall dar. Beachtenswert ist dabei, daß selbst geringe Konzentrationen an Rheumafaktor zur Vortäuschung einer deutlich spezifischen IgM-Antwort ausreichen (Tab. 7). Nach einmaliger, subtotaler Adsorption der Rheumafaktoren sind zunächst falsch angezeigte hohe AntiRubella-IgM-Titer nicht mehr in dieser Höhe nachweisbar. Wie in Tabelle 7 dokumentiert, folgt der Abnahme des Rheumafaktor-Titers konsequent die entsprechende Reduktion des falsch positiven „spezifischen" IgM-Titers.

Auch in ihrer Aussage einfache Teste ohne quantitative Titerangaben wie der Rubazym ${ }^{\circledR}-\mathrm{M}$-Test zeigen eine Rheumafaktor-Interferenz (Abb. 3). Im „Graubereich" dieser Methode sind die erhaltenen Angaben (Absorbanzen) deutlich abhängig von der in der Probe enthaltenen Rheumafaktor-Aktivitäten. Die Adsorptionskapazität unseres Adsorbens war in den von uns untersuchten Proben auch bei Einsatz nicht vorverdünnter Seren immer ausreichend hoch, um die durch Rheumafaktoren vorgetäuschten spezifischen IgM-Titer zu eliminieren. Gleichwohl wäre es theoretisch denkbar, daß bei extrem hohen RheumafaktorKonzentrationen (Rheumafaktor-Titer $>1: 2000$ ) eine zweite Adsorption bzww. eine Vorverdünnung der Proben erforderlich wäre. Da jedoch bei den meisten ELISA-Verfahren zum Nachweis spezifischer IgM-

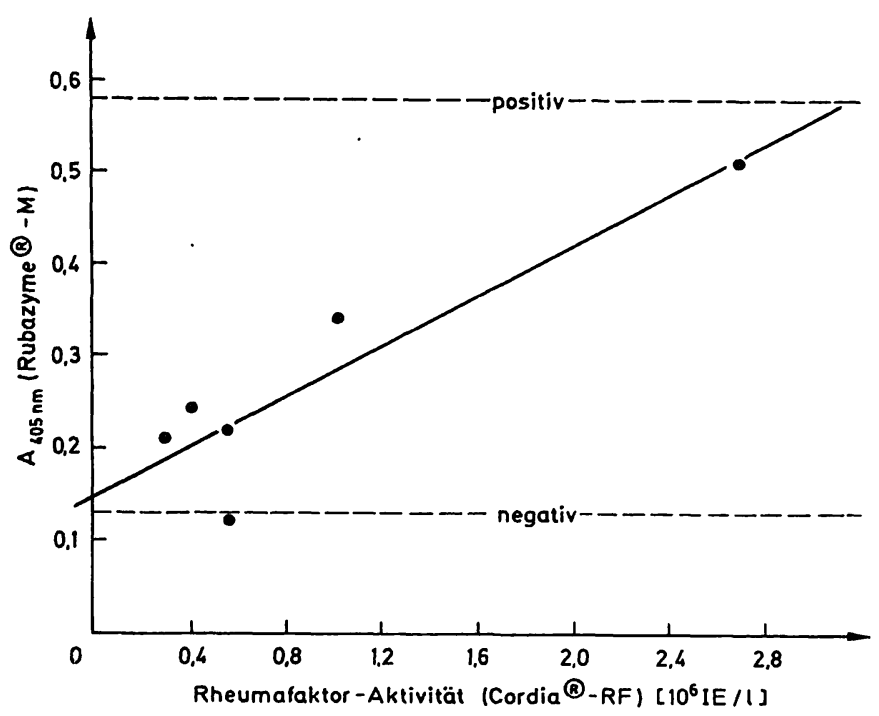

Abb. 3. Abhängigkeit der Indikatorreaktion (Rubazyme ${ }^{\circledR}-M$ ) von der Rheumafaktor-Aktivität (IE), gemessen mit einem Rheumafaktor-ELISA (Cordia ${ }^{\circledR}$-Rheumafaktor).

Antikörper Probenvorverdünnung in der Regel vorgeschrieben sind, böte sich für die Praxis die Einschaltung der Rheumafaktor-Adsorption im Rahmen der Probenverdünnung an, zumal die Adsorptionsleistung unseres Adsorbens in dünnen, d. h. geringer konzentrierten Adsorbenssuspensionen deutlich gesteigert ist (vgl. Abb. 2).

Durch die Rheumafaktor-Adsorption durch unser Adsorbens kommt es nicht zum Verlust spezifischer Antikörper. Entsprechend konnten im Hämagglutinationshemmtest Rube HIT ${ }^{\circledR}$ vor und nach Rheumafaktor-Adsorption keine Titerunterschiede gefunden werden (Tab. 8).

Tab. 7. Vortäuschung spezifischer IgM-Antikörper gegen Rubella-Antigen durch Rheumafaktoren im Enzym-Immunoassay (ELISA - Enzygnost ${ }^{\circledR}$ - Rubella). Gegenübergestellt sind die vor und nach Teiladsorption der Rheumafaktoren ermittelten IgM-Titer gegen Rubella und die korrespondierenden Rheumafaktor-Titer, ermittelt mit der RheumafaktorLatex-Agglutination (Rapi Tex ${ }^{\mathbb{Q}=R h e u m a f a k t o r) .}$

\begin{tabular}{|c|c|c|c|c|c|}
\hline \multirow[t]{2}{*}{ Patienit } & \multirow[t]{2}{*}{ Diagnose } & \multicolumn{2}{|c|}{$\begin{array}{l}\text { Vor } \\
\text { Rheumafaktor-Adsorption }\end{array}$} & \multicolumn{2}{|c|}{$\begin{array}{l}\text { Nach } \\
\text { Rheumfaktor-Adsorption }\end{array}$} \\
\hline & & $\begin{array}{l}\text { Titer: } \\
\text { Rheuma- } \\
\text { faktor }\end{array}$ & $\begin{array}{l}\text { Titer: } \\
\text { Anti- } \\
\text { Rubella-IgM }\end{array}$ & $\begin{array}{l}\text { Titer: } \\
\text { Anti- } \\
\text { Rubella-IgM }\end{array}$ & $\begin{array}{l}\text { Titer: } \\
\text { Rheuma- } \\
\text { faktor }\end{array}$ \\
\hline $\begin{array}{l}\text { GAU } \\
\text { TSCH } \\
\text { BOH } \\
\text { ARN } \\
\text { RU' } \\
\text { REI } \\
\text { WER }\end{array}$ & $\begin{array}{l}\text { Pneumonie } \\
\text { Rheumatoide Arthritis } \\
\text { Rheumatoide Arthritis } \\
\text { Rheumatoide Arthritis } \\
\text { Rheumatoide Arthritis } \\
\text { Hepatitis B } \\
\text { Lebercirrhose }\end{array}$ & $\begin{array}{l}1: 600 \\
1: 320 \\
1: 160 \\
1: 80 \\
1: 60 \\
1: 40 \\
1: 20\end{array}$ & $\begin{array}{l}1: 640 \\
1: 320 \\
1: 160 \\
1: 80 \\
1: 160 \\
1: 160 \\
1: 160\end{array}$ & $\begin{array}{l}1: 80 \\
1: 40 \\
1: 80 \\
1: 40 \\
1: 80 \\
1: 40 \\
1: 80\end{array}$ & $\begin{array}{l}1: 30 \\
1: 30 \\
1: 20 \\
1: 10 \\
1: 5 \\
0 \\
0\end{array}$ \\
\hline
\end{tabular}


Tab. 8. Bestimmung des Immunitätsstatus gegen Rubella im Hämagglutinationshemmtest (Rube HIT ${ }^{\circledR}, F a$. Behringwerke $A G)$ vor und nach Adsorption der Rheumafaktoren.

\begin{tabular}{lll}
\hline Patient & $\begin{array}{l}\text { Titer: Rubella } \text { HIT }^{\circledR} \\
\text { vor } \\
\text { Adsorption }\end{array}$ & $\begin{array}{l}\text { nach } \\
\text { Adsorption }\end{array}$ \\
\hline GAU & $1: 128$ & $1: 128$ \\
TSCHO & $1: 32$ & $1: 32$ \\
BOH & $1: 128$ & $1: 128$ \\
ARN & $1: 164$ & $1: 164$ \\
RƯ & $1: 16$ & $1: 16$ \\
REI & $1: 32$ & $1: 32$ \\
WER & $1: 256$ & $1: 256$ \\
\hline
\end{tabular}

Spezifische IgM-Antikörper werden nicht adsorbiert. Ein entsprechender Beleg wurde durch die Bearbeitung zweier Anti-Rubella-IgM-haltiger Seren erbracht. Serum „SCHAM“ stammt von einem akut an Röteln erkranktem Schulkind, Serum „LIN“ von einer geimpften jungen Frau, beide waren seronegativ (kein Rheumafaktor). Die Probennahme erfolgte jeweils $14 \mathrm{~h}$ nach Aufschießen des Exanthems bzw.

\section{Literatur}

1. Meurmann, O. H. \& Ziola, B. R. (1978) J. Clin. Pathol. 31, 483.

2. Bütler, R. \& Brunner, E. (1974) Vox Sang. 25, 466-469.

3. Ziegelmaier, R. \& Bieker, R. (1982) Laboratoriumsblätter $32,35-38$.

4. Salonen, E. M., Vaheri, A., Suni, J. \& Wager, O. (1981) J. Infect. Dis. $142,250-255$.

5. Geisen, H. P. \& Seelig, H. P. (1979) Lab. Med. 3, 23-26.

6. Zalan, E., Wilson, C. \& Labzoffsky, N. A. (1973) Arch. Ges. Virusforsch. 40, 1971-173.

7. Gordon, M. A. \& Lapa, E. W. (1974) Am. J. Clin. Pathol. $61,488-494$.

8. Yeni, P., Segond, P., Massias, P. \& Pillot, J. (1978) Lancet I, 219-220.

9. Roberts, R., Chabasse, D. \& Hoque, T. P. (1980) Biomed. Express $35,61-65$.

10. Wager, O. \& Lindström, P. (1982) Scand. J. Immunol. 1, $319-328$.

11. Wager, O., Lindström, P., Räsänen, J. A., Kekomäki, R., Ziola, B., Salmi, A., Isomäki, H., Skrifvars, B. \& Penttinen, K. (1982) J. Clin. Exp. Med. 46, 149-160.

12. Sever, J. L. (1962) J. Immunol. 88, 320 zitiert n. Bouin, O. (1973) Quantitativ-virologische Methodik, Stuttgart p. 228.

13. Yolken, R. H. \& Leister, F. J. (1981) J. Clin. Microbiol. 14, $427-432$.

14. Leinikki, P. O., Shekarchi, I., Dorsett, P. \& Sever, J. L. (1978) J. Lab. Clin. Med. 92, 849-857.

15. Edgeskjold, E. M., Graudal, H., Sorensen, H. J. \& Permin, H. (1977) Lancet II, 456-457.

16. Zuraw, B. L., O'Hair, C. H., Vaughan, J. H., Mathison, D. A., Curd, J. G. \& Katz, D. H. (1982) J. Clin. Invest. 68 , 1610-1613.

17. Hrncir, Z., Vavrina, J., Salavec, M. \& Tichy, M. (1976) Rev. Czech. Med. 21, 166-170.

18. Verdier, J. M., Cassuto, J. P., Viot, G., Quaranta, J. F. \& Krebs, B. P. (1981) Nouv. Presse Med. 9, 1973-1075.

19. Silverberg, J., Row, V. V. \& Volpier, R. (1978) Am. Intern. Med. 88, 216-217. post vaccinationem. Wie in Tabelle 9 dokumentiert, hat das Adsorptionsverfahren dabei keinen Einfluß auf die spezifischen Rubella-IgM-Titer. Die im Testansatz mitgeführten Rheumafaktor-haltigen Seren „BICK“ und „LAN" dagegen zeigten, wie zu erwarten, einen deutlichen Titerabfall bzw. eine Elimination der falsch positiven IgM-Titer.

Tab. 9. Kein Einfluß der Rheumafaktor-Adsorption (Adsorbens + Serum $=1+1$ ) auf die anti-Rubella IgMTiter bei SCHAM und LIN; die Rheumafaktor-Seren LAN und BICK zeigen einen deutlichen Titerabfall nach Rheumafaktor-Adsorption. Kleinste Titerstufe 1:40 (d. h. $<1: 40=0$ ), Enzygnost ${ }^{\circledR}$-Rubella.

\begin{tabular}{lll}
\hline Patient & \multicolumn{2}{l}{ Titer: Anti-Rubella-IgM } \\
& $\begin{array}{l}\text { vor } \\
\text { Rheumafaktor- } \\
\text { Adsorption }\end{array}$ & $\begin{array}{l}\text { nach } \\
\text { Rheumafaktor- } \\
\text { Adsorption }\end{array}$ \\
\hline SCHAM & $1: 1280$ & $1: 1280$ \\
LIN & $1: 160$ & $1: 160$ \\
\hline LAN & $1: 160$ & $<1: 40$ \\
BICK & $1: 160$ & $<1: 40$ \\
\hline
\end{tabular}

20. Markenson, J. A., Daniels, C. A., Notkins, A. L., Hofnagle, J. H., Gerety, J. \& Barker, L. F. (1976) Clin. Exp. Immunol. 19, 209-217.

21. State, I., Runcan, V., Voiculescu, M. \& State, D. (1973) Rev. Roum. Med. Intern. 10, 29-36.

22. Kacaki, J. N., Siem, T. H. \& Brouwer, R. (1977) Lancet $I$, $1008-1009$.

23. Roberts-Thomson, P. J. \& Bradley, J. (1980) Clin. Exp. Immunol. 37, 408-415.

24. Capra, J. D., Winchester, R. J. \& Kunkel, H. G. (1969) Arthr. Rheum. 12, 67-73.

25. Cohen, J., Joyce, L., Blanchard, F., Etienne, J. C., Gougeon, J. \& Dropsy, G. E. (1982) Ann. Immunol. 132, $201-210$.

26. Meurmann, O. H., Terho, P. \& Salmi, A. (1979) Lancet II, 685-686.

27. Vejtorp, M. (1979) Lancet $I, 215-216$.

28. Vejtorp, M. (1981) Acta Pathol. Microbiol. Scand. 89, $123-128$.

29. Naot, Y., Barnett, E. V. \& Remington, J. S. (1981) J. Clin. Microbiol. 14, 73-78.

30. Skaug, K. \& Gaarder, P. J. (1978) Acta Pathol. Microbiol. Scand. Sect. C. Immunol. 86, 33-36.

31. Ziegelmaier, R., Bieker, R., Behrens, W. \& Vermeer, H. (1983) Laboratoriumsblätter 33, 19-25.

32. Weichselbaum, T. E. (1946) Am. J. Clin. Pathol. 10, 40. Zitiert n. W. Rick (1977) Klinische Chemie und Mikröskopie Berlin - Heidelberg-New York.

33. Avrameas, S. \& Ternyck, T. (1969) Immunochem. 6, $53-66$.

34. Allen, J. \& Kunkel, H. G. (1966) Arthr. Rheum. 9, $758-768$.

Dr. Dr. Gerald Kolb

Klinikum der

Philipps-Universität Marburg

Zentrum für Innere Medizin

Med. Klinik und Poliklinik

Lahnberge

D=3550 Marburg 